AIAA 2001-1580

Characterizing Aeroelastic Systems using

Eigenanalysis, Explicitly Retaining the

Aerodynamic Degrees of Freedom

Jennifer Heeg

NASA Langley

Hampton, VA

Earl H. Dowell

Duke University

Durham, NC

42 ${ }^{\text {nd }}$ AIAA/ASME/ASCE/AHS/ASC Structures, Structural Dynamics and Materials Conference

$$
\text { 16-19 April } 2001
$$

Seattle, Washington

For permission to copy or to republish, contact the copyright owner named on the first page.

For AIAA-held copyright, write to ALAA Permissions Department, 1801 Alexander Bell Drive, Suite 500, Reston, VA, 20191-4344. 


\title{
Characterizing Aeroelastic Systems using Eigenanalysis, Explicitly Retaining the Aerodynamic Degrees of Freedom
}

\author{
Jennifer Heeg ${ }^{x}$ \\ NASA Langley Research Center \\ Hampton, Virginia \\ Earl H. Dowell ${ }^{\S}$ \\ Duke University \\ Durham, North Carolina
}

\begin{abstract}
Discrete time aeroelastic models with explicitly retained aerodynamic modes have been generated employing a time marching vortex lattice aerodynamic model. This paper presents analytical results from eigenanalysis of these models. The potential of these models to calculate the behavior of modes that represent damped system motion (noncritical modes) in addition to the simple harmonic modes is explored. A typical section with only structural freedom in pitch is examined. The eigenvalues are examined and compared to experimental data. Issues regarding the convergence of the solution with regard to refining the aerodynamic discretization are investigated. Eigenvector behavior is examined; the eigenvector associated with a particular eigenvalue can be viewed as the set of modal participation factors for that particular mode. For the present formulation of the equations of motion, the vorticity for each aerodynamic element appears explicitly as an element of each eigenvector in addition to the structural dynamic generalized coordinates. Thus, modal participation of the aerodynamic degrees of freedom can be assessed in addition to participation of structural degrees of freedom.
\end{abstract}

\section{INTRODUCTION}

A long-standing procedure for examination of structural dynamic systems is to employ eigenanalysis. It is becoming more commonly accepted to utilize the same procedure for examination of aerodynamic systems and aeroelastic systems ${ }^{1 .}{ }^{2}$. In this paper, aeroelastic

\$Aerospace Eng., Aeroelasticity Branch

\$J.A. Jones Professor, Dept of Mechanical Engr and Matl Sci; Fellow AIAA

Copyright (C) 2001 by the American Institute of Aeronautics and Astronautics, Inc. No copyright is asserted in the United States under Title 17, U.S. Code. The U.S. Govemment has a royalty-free license to exercise all rights under the copyright claimed herein for Governmental Purposes. All other rights are reserved by the copyright owner. eigenanalysis results are presented. This work is an extension of previously published work $^{3.4}$ utilizing a finite-wake, time-domain, discretized vortex lattice aerodynamic model, coupled with time-domain discretized structural dynamic, downwash and aerodynamic forcing equations. In the previous publications, results of aerodynamic parametric variations were presented and the properties of the aerodynamic model were examined in detail. In the current work, the aeroelastic eigenanalysis properties are examined from a similar perspective. The results will be discussed first by examination of the eigenvalue behavior, followed by examination of the eigenvectors.

As was pointed out in the previous publications, the modes of the aerodynamic model are artifacts of the discretization of the finite wake length. Because the modes result from the approximations employed, their validity is a function of the chosen discretization scheme. Both the validity of the aeroelastic results and the ease with which one can interpret the system behavior will be shown to depend upon the discretization.

One of the shortcomings of most linear aeroelastic analysis procedures is the inability to calculate the modes of the system when the motion is not simple harmonic ${ }^{5.6}$. That is, under the assumptions of the analysis, the modes of the system are only valid when they are neutrally stable. These linear methods serve well for examining the stability and the frequency of the critical mode at the instability, but provide insufficient information for examining damped (noncritical) system. There are several notable examples in the literature where noncritical mode characteristics have been calculated, employing various aerodynamic modeling techniques, 7.8 . A summary of this work can be found in $\mathrm{Heeg}^{4}$. The current work examines the potential of the eigenanalysis procedure, applied to the discrete time vortex lattice model, to calculate the behavior of modes that represent damped system motion (noncritical modes) in addition to the neutrally stable simple harmonic motion. 
Aeroelastic instabilities are often said to occur because the aerodynamics provide a coupling mechanism for structural dynamic modes 9,10 . In the current work, we examine the aeroelastic eigenvectors and their progression with dynamic pressure. In the case of the model presented, the mode shapes consist of the generalized structural coordinates and the vorticities on each aerodynamic element. The eigenvectors are examined in various ways in attempts to distill essential information regarding the coupling between modes.

A typical section with only a rotational structural degree of freedom (pitch) is analyzed in this paper. The configuration was the subject of a wind tunnel experiment; it is a typical section airfoil with only a rotational structural degree of freedom, which is shown to be torsionally divergent. The experimental results are presented by Heeg in reference 4 .

This paper is organized as follows. A recapitulation of the aerodynamic model is presented. The aeroelastic equations of motion and stability analysis method are reviewed. The aeroelastic eigenvalues of the example configuration are examined and compared with experimental data. Trends in the aeroelastic eigenvalues with variations in the aeroelastic model discretization are discussed. Finally, the information provided by the aeroelastic eigenvectors is examined.

\section{AERODYNAMIC MODEL}

A summary of the aerodynamic model and the characteristics of the eigenvalues are presented here. For a more thorough discussion, the reader is referred to references 3 and 4 . A Vortex Lattice solution to Laplace's equation for incompressible two-dimensional flow is utilized. The flow over an airfoil and in the wake is modeled. The airfoil is represented as a 2 dimensional flat plate. The airfoil and the wake are spatially divided into elements; the vortex lattice kernel function is utilized at a frozen instant in time, and Kelvin's theorem is applied preserving the circulation as time advances. Convection of vorticity in the wake is explicitly modeled; dissipation of the vorticity is included through use of a temporal relaxation factor applied to the last element of the wake mode. Timemarching equations are written, generating a mathematical model of the flow that is both spatially and temporally discretized, Eqn 1.

$$
[A]\{\Gamma\}^{n+1}+[B]\{\Gamma\}^{n}=\{w\}^{n+1} \quad \text { Eqn } 1
$$

Once these equations are written, they inherently contain the approximations of the finite wake and the discretization. Heeg and Dowell ${ }^{3}$ present a detailed study of the effects of the aerodynamic model discretization on the aerodynamic eigenvalues. The size $(\Delta x)$ and number ( $M$ on the wing, $N_{\text {wake }}$ in the wake) of elements, and velocity (U) are related through Eqn 2 and Eqn 3.

$$
\begin{array}{ll}
\Delta x=\frac{2 b}{M} & \text { Eqn } 2 \\
U=\frac{\Delta x}{\Delta t} & \text { Eqn } 3
\end{array}
$$

The time-marching aerodynamic equations are analyzed subject to the boundary condition that the downwash, w, is zero for all time. This is equivalent to the flat plate representation of the wing being at zero angle of attack. The eigenvalues of these uncoupled aerodynamic equations are evaluated by applying a ztransform to the discrete time equations. The discrete time eigenvalues, $z$, are extracted from these transformed equations and converted to the continuous time domain through a zero older hold.

$$
\lambda=\frac{\log (z)}{\Delta t} \quad \operatorname{Eqn} 4
$$

The eigenvectors are also extracted from the timemarching equations. The continuous time eigenvalue distribution for a representative case, with 10 aerodynamic elements on the wing and 90 in the wake, is shown in Figure 3. This plot was generating using a value of 1 for reduced velocity (V), defined in Eqn 5 .

$$
V=\frac{U}{\omega_{\alpha} b} \quad \text { Eqn } 5
$$

Reduced velocity, $\mathrm{V}$, is defined as the velocity normalized by the torsional mode frequency and the semi-chord. The axes scale with velocity, but the pattern does not change. The complex aerodynamic eigenvalues are discretely spaced and arranged in "arms" that emanate from the origin of the continuous time complex plane and reach up and down in the left half plane. Additionally, the real parts of the arms asymptotically approach a limiting value.

The discrete time aerodynamic equations are rank deficient by the number of aerodynamic elements which are placed on the wing, $M$. This produces $M$ discrete time eigenvalues at the origin in the $\mathrm{z}$-domain. Transformation of a pole at origin into continuous time results in a pole at negative infinity along the real axis. The continuous time aerodynamic model thus contains $M$ eigenvalues at $-\infty$ on the real axis. 
For the continuous time eigenvalue distribution, shown in figure 1 , the presence of positive aerodynamic damping is evidenced by the arms lying in the left half plane. Aerodynamic eigenvalues have been shown to be artifacts of the discretization. The uncoupled aerodynamic eigenvalues scale as the inverse of velocity due to the presence of the time step in the denominator of the zero order hold transformation relationship shown in Eqn 4. These aerodynamic eigenvalues exist in the model, even in the case where there is no coupling with a structural model, as exemplified in the above case where the downwash has been set to zero.

In previous work ${ }^{3}$, the following observations were made with regard to the aerodynamic model and the resulting eigenanalysis results. The effects of discretization are controlled by two independent factors. The size of the elements determines the range of frequencies covered by the eigenvalues. The number of elements in the wake drives the damping; increasing the number of complex aerodynamic modes moves the eigenvalue arms closer to the imaginary axis. The effects of size and number are shown to be independent, as one controls the transformation from discrete to continuous time, and the other controls the discrete time eigenvalue pattern. The effect of the discretized finite wake is to produce discretely spaced eigenvalues, instead of a continuous line. As the wake length is increased, the eigenvalues become more closely spaced; the frequency spacing is approximated using Eqn 6.

$$
\Delta \omega \cong \frac{2 \pi U}{L_{\text {wake }}} \quad \text { Eqn } 6
$$

An aerodynamic eigenvector contains the vorticity for each aerodynamic element associated with that mode. Four aerodynamic eigenvectors are represented in Figure 4. These "modal vorticities" are plotted as functions of the chord-wise location of the associated aerodynamic elements, normalized by the chordlength and referenced to the wing mid-chord. The first mode, a real mode, resembles a static pressure coefficient distribution over the wing, with little participation from the wake. The second mode is also a real mode resembling a static pressure distribution over the wing, but with significant wake participation. The remaining aerodynamic modes are complex and are comprised primarily of an oscillating wake. The wing vorticities are insignificant compared to those in the wake. The modes are presented in order of increasing frequency. Each mode contains a single frequency; as the frequency increases or as the mode number is advanced more oscillations are observed in the wake.
For each of the presented eigenvectors, the amplitude of the oscillations becomes larger toward the end of the wake. Edwards presents vorticity distributions for stable and unstable oscillatory modes in reference 8. The eigenvectors can be thought of as time histories of vorticity, where time progresses from right to left. As Edwards points out, a snapshot of vorticity where the amplitude is large at the end of the wake indicates a stable motion. Large amounts of vorticity were shed from the wing initially; that vorticity has propagated downstream. Smaller amounts of vorticity shed from the wing as time advances.

A subtlety regarding the aerodynamic eigenvectors is their variance with reduced velocity. The effect of doubling the reduced velocity is to halve the time period represented by each vorticity snapshot and thereby double the frequency associated with that eigenvector. There are two points to be made: 1) the eigenvectors, which represent spatial distributions of vorticity, are invariant with the reduced velocity, but 2) the period of time represented by the vorticity snapshot changes with reduced velocity. These points follow from previous work, where it was shown that the eigenvectors of the continuous and discrete time systems are identical ${ }^{4}$. The eigenvector associated with a mode, whether it is in discrete or continuous time, is a spatial distribution of vorticity, as exemplified by Figure 4 . The reduced velocity does not appear in the discrete time aerodynamic equations; these equations and their eigensolution are applicable at all reduced velocities. The influence of the reduced velocity appears in analysis of the continuous time system because the time step size appears in the denominator of the transformation law given in Eqn 4. The time period over which the vorticity convection snapshot occurs is the number of wake elements multiplied by the time step size. Rearrangement of Eqn 2, Eqn 3 and Eqn 5 shows that the time step size is proportional to the inverse of reduced velocity. Thus, doubling the reduced velocity halves the time step size, halving the time period of the vorticity snapshot, doubling the frequency associated with that eigenvector. So, although the eigenvector is invariant with reduced velocity, interpretation of the information does change.

\section{AEROELASTIC MODEL}

Attention is now turned to examining the coupled aeroelastic system. The aerodynamic equations discussed previously are now considered in conjunction with a time-marching model of the structural dynamic behavior. The interactions between structural motion and aerodynamic forces are incorporated through two fundamental relationships: structural loads due to aerodynamics are produced by 
unsteady vorticity on the wing; downwash is induced on the airfoil by the structural motion. Combining these relationships produces the governing equation for an aeroelastic system, Eqn 7, where $q$ is the vector of generalized structural coordinates.

$$
\left[Q_{2}\right\}\left\{\begin{array}{l}
q \\
\Gamma
\end{array}\right\}^{n+1}+\left[Q_{1}\right]\left\{\begin{array}{l}
q \\
\Gamma
\end{array}\right\}^{n}=\left\{\begin{array}{l}
0 \\
0
\end{array}\right\} E q n 7
$$

A simplified aeroelastic system is utilized in this study. The typical section, Figure 5, is a structural and aerodynamic idealization where its motion and the airflow about it can be represented as two-dimensional. The airfoil section is considered rigid and its permitted motion limited to rotation about a fixed axis. This axis of rotation is termed the elastic axis; its position, denoted $e$, is measured positive aft from the center of pressure. The structural dynamic stiffness of each mode is represented by a linear spring constant. The geometric parameters are illustrated in Figure 5; the non-dimensional parameters of interest are defined Table 1. Table 1 also provides the numerical values of the parameters for the typical section to be analyzed.

\section{AEROELASTIC EIGENVALUES}

The stability of the aeroelastic system was determined through eigenanalysis of the equations of motion for a series of reduced velocities. The initial stability analysis was performed using an aerodynamic discretization with 10 elements on the wing and 90 elements in the wake; the resultant continuous time root locus is shown in Figure 6. For clarity, only the region near the origin is presented. The figure shows the eigenvalues progressing as the reduced velocity is increased. There are many additional complex modes which originate in the aerodynamics, which are off the scale of the plot. There are also eigenvalues at negative infinity, not shown in the figure, the number of which is the same as the number of aerodynamic elements on the wing.

This configuration destabilizes at zero frequency, termed divergence. The eigenvalues at the divergence reduced velocity, 3.8 , are distinguished in the figure by square symbols. This analysis indicates that the dynamic mode still exists with a nonzero frequency when the system becomes unstable. At this velocity, the dynamic mode is a coupled structural and aerodynamic mode; the modal content and resultant system behavior will be addressed subsequently.

From the aeroelastic root locus, the complex primarily aerodynamic modes can be readily identified. The uncoupled aerodynamic eigenvalues were presented in
Figure 3, for a reduced velocity of 1.0. As velocity increases, these eigenvalues migrate along lines of constant damping. Several lines of constant damping have been superimposed on the aeroelastic root locus, Figure 6, to illustrate this point. Although all modes shown in the aeroelastic root locus belong to the coupled system, there is relatively little influence of the structure on what originated as complex aerodynamic modes. This feature will be examined further in terms of the aerodynamic parameterization and through eigenvector characteristics.

Increasing velocity produces a migration in the structural dynamic (in this case, pitch) mode also. The coupled mode that originates as the structural dynamic mode will be referred to here as simply the dynamic mode of the system. This mode is a pure structural mode only at zero airspeed. For any non-zero velocity, it and all other modes are strictly speaking aeroelastic modes. The lowest reduced velocity for which this system was analyzed was 0.1 . The dynamic mode for this nearly-zero velocity is indicated by a solid triangle in Figure 6 at $49.5 \mathrm{rads} / \mathrm{second}$, the torsional natural frequency. Increasing velocity produces an ever larger aerodynamic effect on the on the pitch mode. This aeroelastic coupling causes the dynamic mode frequency to decrease as velocity increases. This trend holds true until the system becomes unstable at a reduced velocity of 3.8 , when a zero frequency root, aerodynamic in origin, migrates across the imaginary axis. Once the static mode destabilizes, the frequency of the dynamic root, structural in origin, no longer tends toward zero.

Because the system diverges, the real roots, aerodynamic in origin, are of primary concern. Two real poles originate from the present aerodynamic model. For increasing airspeed, both roots initially become more stable. However, at approximately $75 \%$ of the divergence reduced velocity, one root changes direction and eventually destabilizes. The mechanism responsible for the divergence of this aeroelastic model has its origin in the aerodynamic terms.

A wind tunnel test of this typical section configuration was conducted by Heeg ${ }^{4}$. Figure 5 shows experimental data for the dynamic mode, plotted with the analytical results. Good agreement was obtained, with regard to the frequency of the dynamic mode. The damping comparison does not fare as well.

\section{AERODYNAMIC PARAMETRIC VARIATIONS}

\section{Variation of wake length, maintaining element size}


Discrete aerodynamic eigenvalues arise in this model because finite approximations are utilized. The aerodynamic model contains complex modes, with different frequency and damping, dependent upon the discretization. The aerodynamic model also contains modes with eigenvalues at zero in the discrete time domain, or at negative infinity in the continuous time domain. These aerodynamic model characteristics, which are direct results of the discretization chosen, strongly affect the eigenvalues associated with the primary aeroelastic modes.

A brief study is presented to address the effects of discretization on convergence and modal identity. Different aerodynamic discretizations are used to generate aeroelastic models. The eigenvalues of the uncoupled aerodynamic equations are compared to the eigenvalues produced by the coupled aeroelastic equations.

The first effect to be examined is the influence of the number of elements in the wake, given a fixed number of elements on the wing. Thus, for this set of variations, the computational domain is extended while the element size is maintained. All cases for this parametric variation contain 10 elements on the wing. The wing semi-chord is 4 inches, producing a spatial element size of 0.8 inches from Eqn 2. The analyses were conducted at the divergence reduced velocity, 3.8, resulting in the time step size of 0.001063 seconds from Eqn 3 and Eqn 5. The aerodynamic and aeroelastic eigenvalues that result from four discretizations are presented in Figure 8. The four discretization cases shown contain 90, 270, 360 and 1440 wake elements, producing wake lengths of 9,27 , 36 and 144 wing chord lengths respectively. In the figure, the eigenvalues of the uncoupled aerodynamic system are plotted as open symbols; the eigenvalues of the coupled aeroelastic system are plotted as solid symbols. Figure 8 is now discussed in detail.

The discretization case with 90 elements in the wake is discussed first. In Figure 8, the eigenvalues for this case are represented by circles. With 90 elements in the wake, the complex aeroelastic eigenvalues are nearly identical to complex aerodynamic eigenvalues, as shown by the solid circles and the open circles lying on top of each other. The exception to this statement is the presence of the aeroelastic mode, indicated by the solid circle at $-16.4+\mathrm{j} 26.3$, which does not appear in the uncoupled aerodynamic system. This mode corresponds to the mode which originated as the structural dynamic mode also seen in Figure 6, which presents the variation with reduced velocity for this discretization.
In Figure 8 , the aerodynamic and aeroelastic eigenvalues lying on the real axis differ significantly from each other. For this discretization case, there are 2 real aerodynamic eigenvalues present, indicated by open circles on the real axis at -3.5 and -34.6 . The real aeroelastic eigenvalues are at the origin, indicating divergence of the system, and at -10.1 . The difference between the aerodynamic and aeroelastic eigenvalues is indicative of coupling between the structure and the aerodynamics.

Thus, with the 90-element-wake discretization of the aerodynamic model, identification of the primary aeroelastic modes is straightforward. This analysis indicates that there are three modes which are affected significantly by the coupling between the aerodynamics and the structural dynamics: the structural-dynamic-originated mode, the static divergence mode and a static stable mode. Further evidence of this "modal clarity" will be provided in the discussion of the eigenvectors later in this paper.

Consider now the case where the number of elements in the wake is 270 . This data is shown in Figure 8 by the square symbols. Because there are more aerodynamic elements in the model, there are more aerodynamic modes present in the system. Recall from the discussion of the aerodynamic parametric variations that an increase in the number of complex aerodynamic modes moves the aerodynamic eigenvalue arms closer to the imaginary axis. Also from that discussion, recall that increasing the wake length results in an increase in the modal density. Both of these trends are observed in Figure 8 by comparing the 270 element case to the 90 element case.

The complex aeroelastic eigenvalues associated with the 270 element discretization are nearly identical to the complex aerodynamic eigenvalues, as was observed in the 90 element case. Again, the presence of a complex aeroelastic eigenvalue in excess of the complex aerodynamic eigenvalues signals the presence of the dynamic aeroelastic mode that originated as the structural dynamic mode, indicated by the solid square at $-14.4+\mathrm{j} 24.9$. Through comparison with the 90 element case, it is seen that increasing the number of elements in the wake causes the complex aeroelastic eigenvalue to shift slightly. Also, the next higher frequency aeroelastic eigenvalue is slightly separated from the nearest aerodynamic pole. The difference is small, but the solid and open squares, with imaginary parts near $30 \mathrm{rads} / \mathrm{sec}$, do not overplot. This difference signals the onset of "modal obscurity." Modal obscurity is defined by the structural dynamic originated eigenvalue no longer being clearly distringuishable from the complex primarily 
aerodynamic eigenvalues of the aeroelastic system. Concerning the real eigenvalues, there are now 3 real aerodynamic poles, and also 3 real aeroelastic poles. Aeroelastically, they show the divergent mode, and two additional static modes.

As the number of elements in the wake increases, more of the aeroelastic eigenvalues deviate from the aerodynamic eigenvalues. The lower frequency eigenvalues differ by larger and larger amounts from those for the discretizations with fewer wake elements. Thus, the certainty with which a structural dynamic participating mode can be identified diminishes and, ultimately, disappears. This is counter-intuitive. The irony here is that as more elements are added to the wake, presumably to improve the approximations, the less clarity there is regarding the individual components from which the model is comprised.

The case where there are 360 elements in the wake demonstrates modal obscurity effectively. In this case, there are two complex eigenvalues of the aeroelastic system $(-12.7+\mathrm{j} 22.2$ and $-16.1+\mathrm{j} 31.1)$ in the vicinity of the structural-dynamic originated eigenvalues generated by the previously discussed discretizations. Both of these eigenvalues are fairly well separated from any aerodynamic poles. Additionally, the number of complex aeroelastic eigenvalues is now equal to the number of complex aerodynamic poles. Neither of these eigenvalues can be said to be the structural dynamic participating mode.

Further increasing the number of elements in the wake, to 1440 , produces an even more modally obscure picture. The data associated with this discretization is shown in Figure 8 and reproduced on an expanded scale in Figure 9. This plot shows that many of the aeroelastic eigenvalues deviate significantly from the aerodynamic eigenvalues. It is thus impossible from this plot to identify a single mode which is representative of the structural dynamic damping and frequency. From this plot, it is also evident that the eigenvalues become quirky at the uncoupled structural dynamic frequency, $49.5 \mathrm{rads} / \mathrm{sec}$, even though this model is constructed at the divergence reduced velocity, 3.8. At a velocity this high, coupled system behavior would not be expected to appear at the air-off natural frequency. The source of this characteristic has not been identified to date.

Variation of aerodynamic element size, maintaining wake length

A second set of parametric variations is now presented. Figure 10 shows aerodynamic and aeroelastic eigenvalues that result from four new discretizations of the aerodynamic system. In these cases, the wake length has been held constant at 9 chord lengths while the number of wake and wing elements increase. This set of parametric variations results in an increasingly finer aerodynamic grid with increasing number of elements, while holding the computational domain at a fixed size. The number of elements on the wing controls the spatial and temporal discretizations, Eqn 2, Eqn 3 and Eqn 5. The spatial and time step sizes thus vary among the different discretization cases, becoming smaller as the number of elements increases. The four discretization cases shown contain 90,270, 360 and 1440 wake elements. Given that the wake length is held constant at 9 wing chords, the wing is partitioned such that the number of elements on the wing, $M$, equals $10,30,40$ and 160 elements respectively. The eigenvalues for these variations are again presented for a single velocity, corresponding to the divergence reduced velocity, 3.8. In the figure, the eigenvalues of the uncoupled aerodynamic system are plotted as open symbols; the eigenvalues of the coupled aeroelastic system are plotted as solid symbols.

Examining the eigenvalues in Figure 10, it does not at first appear that the complex aerodynamic eigenvalue arms follow a logical progression with increasing number of elements. It turns out, however, that there are two competing changes to the model, which result in this eigenvalue distribution. Recall that the model has been formulated in the discrete time domain, that is, the equations of motion were written at distinct time steps. The discrete time eigenvalues, $\mathrm{z}$, are computed and then transformed by a zero order hold into the continuous time domain. The zero order hold transformation law is given by Eqn 4 .

When the eigenanalysis of the equations is performed in the discrete time domain, the damping of the eigenvalues monotonically decreases as elements are added to the wake. That is, the magnitudes of the discrete time eigenvalues, $z$, increase towards unity. The corresponding values of the logarithm of $z$ (the numerator of Eqn 2) decrease in magnitude. As elements are added to the wing, the time step size (the denominator of Eqn 2) decreases. The overall effect is that increasing the number of elements initially makes the continuous time domain eigenvalues more highly damped, and then as the model is further discretized, the eigenvalues become more lightly damped. These effects are present for both the eigenvalues that originate as aerodynamic modes and the eigenvalue which originates in the structural dynamic model. To see how these effects manifest themselves for the structural dynamic participating mode, a blow up of this region of the root locus is shown in Figure 11. 
The structural dynamic originated eigenvalue varies somewhat with the discretization, but for the number of elements chosen for examination, it is always clear which eigenvalue primarily captures this behavior. The aeroelastic eigenvalues are much closer to the aerodynamic eigenvalues than shown in the previous cases.

\section{Interpreting the analysis}

The following speculations are offered with regard to the parametric variations presented. While the static aeroelastic characteristics are well captured, even with very coarse discretizations, the dynamic (complex) characteristics require refinement of the wing elements.

The eigenvalue that originated in the structural dynamic mode tends to be the most physically significant of the complex modes. Despite the aerodynamic model eigenvalues being artifacts of the discretization, the coupled aeroelastic model produces a destabilizing static mode and a dynamic mode that represent the physical system. It is anticipated that the structural response will be characterized by the frequency and damping of the dynamic mode eigenvalue when subjected to the aerodynamics at this reduced velocity.

For the cases where the wake lengths and the numbers of elements in the wake are small, the dynamic mode is fairly easily identified. Increasing the length of the wake by adding aerodynamic elements produces eigenvalues where several of the modes appear to capture portions of the structural dynamic behavior. For cases with many wake elements, exemplified by the 10 wing element and 1440 wake element case, the structural dynamic originated mode does not appear separately from the aerodynamic originated poles. It has been effectively obscured among the other aeroelastic eigenvalues.

Holding the wake length constant at 9 chords and increasing the number of elements in the wake produced a much different result. For all discretizations considered, no modal obscuring occurred. Through additional parametric variations, it has been observed that as the wake length gets longer, the modal obscurity creeps in at a lower number of elements.

Data from the first set of variations can be compared to the data from the second set. Consider the cases where there are 1440 elements in the wake. These cases with 10 and 160 elements on the wing appear to have significantly different characteristics. The comparison is a little deceptive, because the frequencies associated with the eigenvalues are very different. The damping is controlled by the number of elements in the wake. A line of constant damping, which passes through the ith eigenvalue for the $M=10$ case will also pass through the ith eigenvalue for the $M=160$ case. However, it will be at a much larger frequency. With regard to the effect on the structural dynamic originated eigenvalue, it appears the damping of the aerodynamic eigenvalues within the vicinity of the aeroelastic mode is the important factor.

Interpreting Figure 8 and Figure 10 together leads to the idea that convergence and modal clarity may be an issue of modal proximity. If the aerodynamic modes near the structure-originated aeroelastic mode frequency are lightly damped, then the structurally originated mode is obscured; its effects get smeared among several or many modes.

These studies were concluded in an unsatisfying way. It cannot be stated that the aeroelastic eigenvalues have definitively converged. Neither have the specific causes of the modal obscurity been identified. Potential sources of numerical errors due to computer round off and approximations in the discrete time tranformation have been investigated, but the sources of these behaviors have not been identified.

\section{AEROELASTIC EIGENVECTORS}

The eigenvector associated with a particular eigenvalue can be viewed as the set of modal participation factors for that mode. The eigenvector components are the degrees of freedom specified in the equations of motion. Here, the equations of motion were formulated such that the degrees of freedom are the structural dynamic generalized coordinates ( $\alpha$ and $\dot{\alpha}$ ) and the vorticity on each aerodynamic element $\left(\gamma_{j}\right.$, for $\mathrm{j}=1,2, \ldots,(\mathrm{M}+\mathrm{Nwake}))$.

Before proceeding to examination of the eigenvectors, we consider how they should be scaled. While the components of a given eigenvector have constant magnitude and phasing relative to the other components of that eigenvector, each eigenvector is unique only to within multiplication by a complex number. It is desirable to be able to examine the similarities and differences among modes and at different velocities. To enable this examination, the arbitrariness must be removed. In this analysis, each eigenvector is normalized such that it has magnitude of 1 , and the phase of all components is relative to the first displacement generalized coordinate. That is, the generalized displacement of the structural degree of freedom (pitch) is a real, positive number for every mode for every velocity. 
The aeroelastic eigenvectors are studied from several perspectives. In past work, direct examination of the modal vorticity has been presented. ${ }^{1}$ In this paper, two different ways of examining the eigenvectors are discussed. The first approach taken is to examine the scalar products produced by subsets of the eigenvector components. Trends in these scalars are examined for different discretizations and as airspeed changes. Secondly, dynamic and static modal moments are then calculated and discussed.

\section{Scalar Products of Eigenvector Component Subsets}

Structural Dynamic Portion of All Modes For this configuration, the structural dynamic portion of each eigenvector consists of one generalized displacement and one generalized velocity. In this case, these are the pitch angle, $\alpha$, and pitch rate, $\dot{\alpha}$. As discussed above, the eigenvectors have been scaled such that the phase of the generalized displacement, $\alpha$, is zero. The generalized displacement thus will always be a positive real value for every mode for every velocity. For this simple system, the generalized velocity component for any eigenvector is related directly to the generalized displacement by the eigenvalue.

$$
\dot{\alpha}_{i}=\lambda_{i} \alpha_{i} \quad \text { Eqn } 8
$$

Thus, knowledge of the structural dynamic portion of any eigenvector allows direct calculation of the corresponding eigenvalue. Alternatively, knowledge of an eigenvalue provides instant knowledge of the relative magnitude and phasing of $\alpha$ and $\dot{\alpha}$.

As an example, a mode that has a purely imaginary eigenvalue has a velocity component which lags the displacement by $90^{\circ}$. Phrased in a different way, the velocity component will be purely imaginary since the displacement component has been restricted to be purely real.

In an attempt to discern the importance of the structural participation in each mode, the magnitude of the modal participation of the structural portion, $\mathrm{MP}_{S}$, of the eigenvectors is calculated. This is done by forming the scalar or inner product for each mode as specified in Eqn 9. In this equation, $H$ indicates the Hermitian (complex conjugate transpose) of the vector.

$$
\boldsymbol{M P}_{S}=\left\{\begin{array}{c}
\alpha \\
\dot{\alpha}
\end{array}\right\}^{\boldsymbol{H}}\left\{\begin{array}{c}
\alpha \\
\dot{\alpha}
\end{array}\right\} \quad \text { Eqn } 9
$$

This particular criterion is examined at the divergence reduced velocity for several of the aerodynamic discretizations analyzed earlier. Three discretizations were chosen to revisit. The magnitudes of the structural participation are plotted in Figure 12 as function of the modal frequency.

The first discretization contains 10 aerodynamic elements on the wing and 90 in the wake. It was previously asserted that the structural participating modes could be identified by simply comparing the aeroelastic eigenvalues with the aerodynamic eigenvalues. The modes which differed significantly were those in which the aeroelastic coupling was important and for which motion of the structure would be anticipated. Examining the structural modal participation, Figure 12a, only one mode contains significant structural participation, identifying it as the primary dynamic mode associated with motion of the structure.

The second discretization contain 40 elements on the wing and 360 elements in the wake. Recall from the eigenvalue distribution shown in Figure 6, that two of the aeroelastic modes were shown to deviate significantly from the aerodynamic modes. The plot of the structural participation in Figure 12b shows that both of these modes contain structural participation. The divergent mode's structural participation is not shown in the plot; it is significantly larger than the participation of the dynamic modes. The stable static mode is labeled in the figure as such.

The third discretization contains 160 elements on the wing and 1440 elements in the wake. The modal obscurity that existed in the eigenvalues is reinforced in the eigenvector characteristics in Figure 12c. The structural participation is smeared among many modes, rather than pointing to a single mode which captures the dynamic mode of the structural motion. Again, the divergent mode is not shown and the stable static mode is labeled.

Examining the magnitude of the modal contributions from the structural components separates the modes with significant aeroelastic coupling from those that remain primarily aerodynamic. This same information can be extracted by examining the portion of the eigenvector containing vorticity on the wing.

\section{Modal Moment Comparison}

The configuration being analyzed destabilizes in divergence. Divergence is classically explained as occurring when the static aerodynamic moment exceeds the static structural restorative moment that can be produced. The structural and aerodynamic moments associated with individual modes can be calculated by using the eigenvector information. The 
static structural modal moments, $\mathbf{M}_{\mathrm{S}}$, corresponding to each mode, denoted $i$, were calculated using the static moment equation and the modal results for angular displacement, Eqn 10.

$$
M_{S_{i}}=K_{\alpha} \alpha_{i} \quad \text { Eqn } 10
$$

The static aerodynamic modal moments, $M_{A}$, are calculated in similar fashion, employing the static aerodynamic equation, Eqn 11.

$$
M_{S_{i}}=\bar{q} \operatorname{SeC}_{L_{\alpha}}\left(\alpha_{i}+\alpha_{\text {rigid }}\right) \quad \text { Eqn } 11
$$

The results from these calculations are compared in Figure 13.

In this comparison, the pitch angle component of each eigenvector was employed. This was the pitch angle, $\alpha_{i}$, used in computing both the structural and the aerodynamic modal moments, Eqn 10 and Eqn 11 . The aerodynamic moment is a function of the total angular rotation of the airfoil, not just the elastic portion of the deflection. The structural moment is a function of only the elastic portion of the deflection. By applying the same angle in both calculations, the aeroelastic amplification factor is in evidence and explains the difference between the calculated static structural and static aerodynamic moments. For each velocity, the ratio of the aerodynamic to structural moment is a ratio of dynamic pressure to divergence dynamic pressure.

The difference between the dynamic moment and the static moments are now discussed. The fundamental difference is that the static moments neglect all time derivatives; the rate term of the structural dynamic freedoms, $\dot{\alpha}$, is neglected. The aeroelastic equations of motion were formulated by setting the dynamic moment (inclusive of time derivative terms, and expressed in terms of the vorticities) produced by the aerodynamics equal to the dynamic moment acting on the structure (inclusive of time derivative terms). The dynamic equality holds for each mode individually, or for the summation of modes. Thus, for a specified mode, the dynamic modal moments are equal for the aerodynamic and structural calculations.

The dynamic modal moments associated with the divergent mode and the dynamic aeroelastic mode are compared to the static modal moments in the figure. The difference between the static and dynamic moments, which results directly from exclusion of the time derivatives in the static calculations, is the oscillatory portion of the motion. Examining the dynamic aeroelastic mode first, it is observed that there is a large oscillatory component, subcritically. This is illustrated by the large difference between the static structural moment and the dynamic moment for the same mode.

Another significant difference between the static moment and the dynamic moment occurs supercritically, observing the divergent mode. The static equations enforce a neutral stability assumption. Because the static mode is unstable, there is a large difference between the moments calculated from the static and dynamic equations.

When a mode is neutrally stable, the motion is simple harmonic and the static and dynamic modal moments agree. This is observed by examining the divergence mode at the divergence reduced velocity, 3.8 , and examining the dynamic mode at zero reduced velocity.

\section{CONCLUDING REMARKS}

The formulation of the aeroelastic equations of motion as described herein has provided eigenvalues associated of the behavior of modes that represent damped system motion (noncritical modes) in addition to neutrally stable motion. This formulation has been shown to be statically consistent as the aerodynamic discretization is refined, but not consistent in terms of the dynamic properties. The dynamic results did not converge as the aerodynamic discretization was refined and in fact, the identity of the modes became obscured.

The modal identity was investigated by consideration of both the eigenvalues and the eigenvectors. The aeroelastic eigenvalues were compared to the aerodynamic eigenvalues. Using a well-behaved discretization produces an eigenvalue pattern where very few modes of the aeroelastic system differ from the modes of the aerodynamic system. The ones that differ significantly were identified as the primary aeroelastic modes. The eigenvectors provide quantitative information which conveys this same message. Examining the magnitude of the modal contributions from the structural components separates the modes with significant aeroelastic coupling from those that remain primarily aerodynamic.

Eigenvector behavior was examined. Information obtained from the eigenvalues and eigenvectors can be used to complement each other. The eigenvectors can be used to discern the information that is contained in the eigenvalues, and can provide information with regard to modal content of the aeroelastic modes.

\section{REFERENCES}

[1] Dowell, Earl H., Kenneth C. Hall, and Michael C. Romanowski. Eigenmode analysis in unsteady 
aerodynamics: reduced order models. Applied Mechanics Review Vol. 50, \#6, p371-385, June 1997.

[2] Hall, Kenneth C. Eigenanalysis of Unsteady Flows about Airfoils, Cascades and Wings. AIAA Journal Vol. 32, No. 12, December 1994.

[3] Heeg, Jennifer and Dowell, Earl H. Aerodynamic and Aeroelastic Insights Using Eigenanalysis. AIAA-99-1473. Presented at the $40^{\text {th }}$ Structures, Structural Dynamics and Materials Conference, St. Louis, Missouri, April 1999.

[4] Heeg, Jennifer. Dynamic Investigation of Static Divergence: Analysis and Testing. $\mathrm{PhD}$ Dissertation, Duke University, Durham, North Carolina, May 2000. (Also published as NASA/TP-2000-210310, November 2000).

[5] Nissim, E. Flutter Analysis Using a New Complex p-Method. Presented at the Confederation of European Aerospace Societies (CEAS) International Forum on Aeroelasticity, Rome, June 1997.

[6] Hassig, Hermann J. An Approximate True Damping Solution of the Flutter Equation by Determinant Iteration. Journal of Aircraft, vol. 8, no. 11, November 1971.

[7] Rodden, William P., and E. Dean Bellinger. Aerodynamic Lag Functions, Divergence, and the British Flutter Method. Journal of Aircraft, vol. 19, no. 7 (July 1982)

[8] Edwards, John E., Unsteady Aerodynamic Modeling and Active Aeroelastic Control. SUDAAR 504, February 1977, Stanford University, Center for systems research.

[9] Bisplinghoff, R.E., Holt Ashley, Roger L. Halfman. Aeroelasticity. Mineola, New York: Dover Publications, 1996.

[10] Dowell, Earl H., Edward F. Crawley, Howard C. Curtiss Jr, David A. Peters, Robert H. Scanlan and Fernando Sisto. A Modern Course in Aeroelasticity. Dordrecht, the Netherlands: Kluwer 1995.

\begin{tabular}{|l|c|c|c|}
\hline \multicolumn{1}{|c|}{ Quantity } & Symbol & Definition & Value \\
\hline $\begin{array}{l}\text { Pitch mode } \\
\text { frequency } \\
\text { (rads/sec) }\end{array}$ & $\omega_{\alpha}$ & $\sqrt{K_{\alpha} / s_{\alpha}}$ & 49.5 \\
\hline $\begin{array}{l}\text { Nondimensional } \\
\text { distance from } \\
\text { aerodynamic } \\
\text { center to elastic } \\
\text { axis) }\end{array}$ & $\mathrm{e} / \mathrm{b}$ & $\mathrm{e} / \mathrm{b}$ & 0.375 \\
\hline \begin{tabular}{l} 
Mass ratio \\
\hline $\begin{array}{l}\text { Non- } \\
\text { dimensional } \\
\text { radius of } \\
\text { gyration }\end{array}$
\end{tabular} & $\mu$ & $\mathrm{m} /\left(1 \rho_{\text {air }} \mathrm{b}^{2}\right)$ & 23.1 \\
\hline $\begin{array}{l}\text { Semichord } \\
\text { (inches) }\end{array}$ & $\mathrm{b}$ & $\sqrt{I_{\alpha} / m^{2}}$ & 0.41 \\
\hline Span (inches) & 1 & & 21 \\
\hline
\end{tabular}

Table 1 Parameters of typical section 


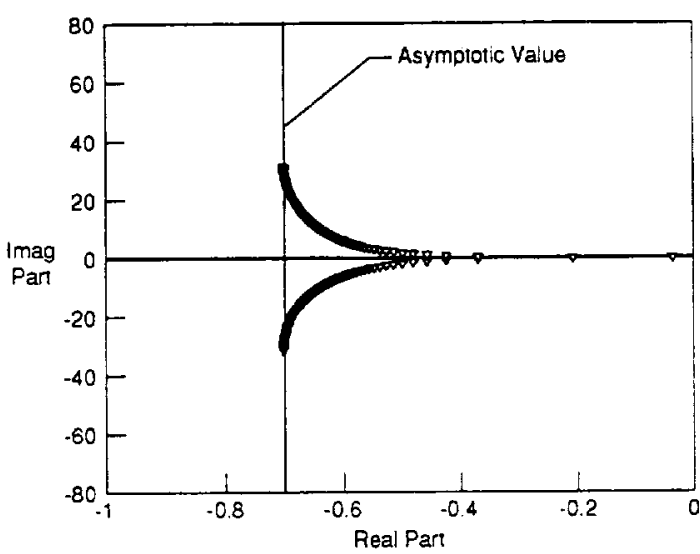

Figure 1 Continuous time aerodynamic eigenvalue pattern
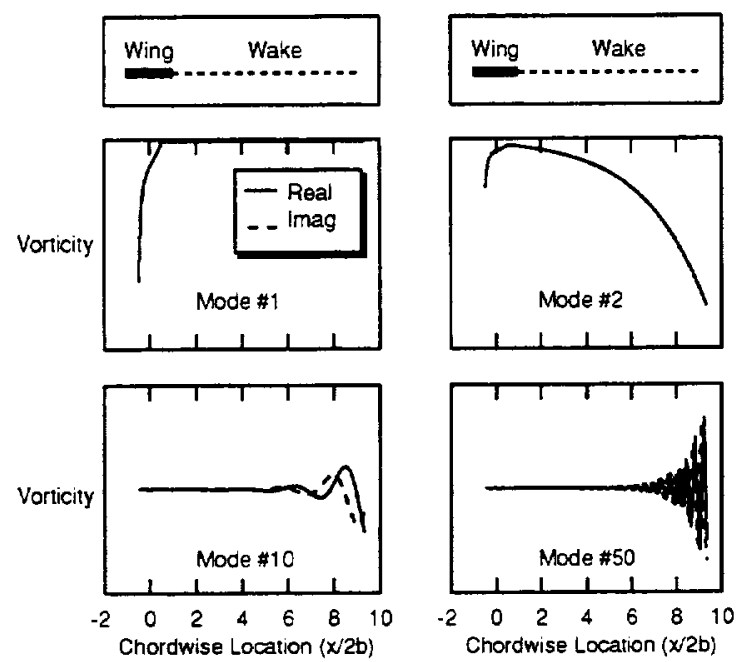

Figure 2 Selected aerodynamic eigenmodes

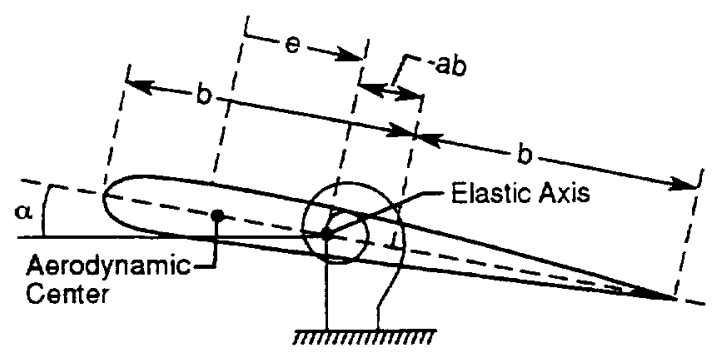

Figure 3 Typical section with pitch freedom

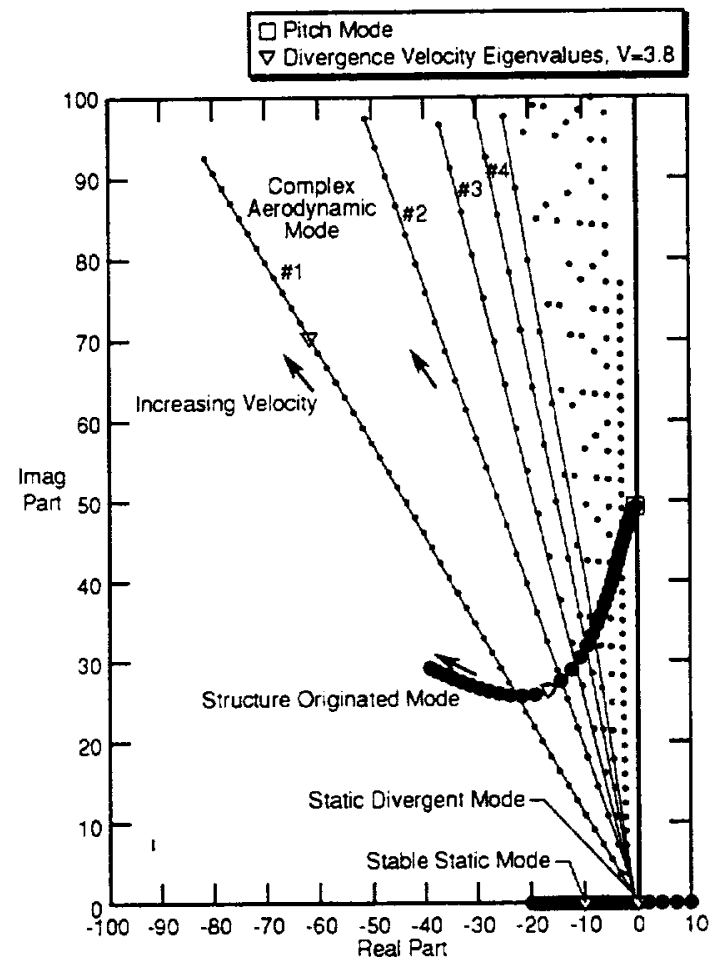

Figure 4 Continuous time aeroelastic root locus
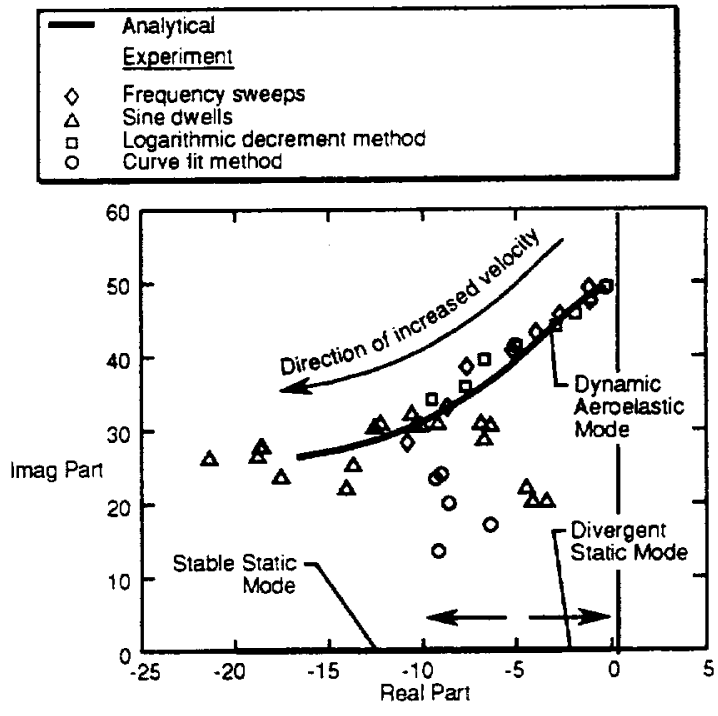

Figure 5 Comparison with experimental data

American Institute of Aeronautics and Astronautics 

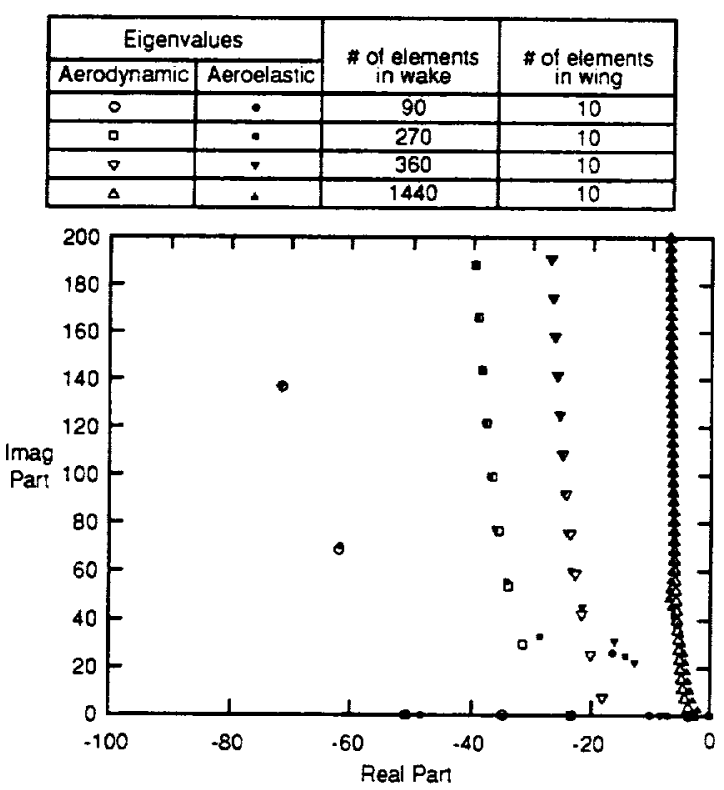

Figure 6 Eigenvalue patterns associated with aerodynamic discretizations with constant number of elements on the wing (constant spatial and temporal discretizations) analyzed at divergence reduced velocity, 3.8

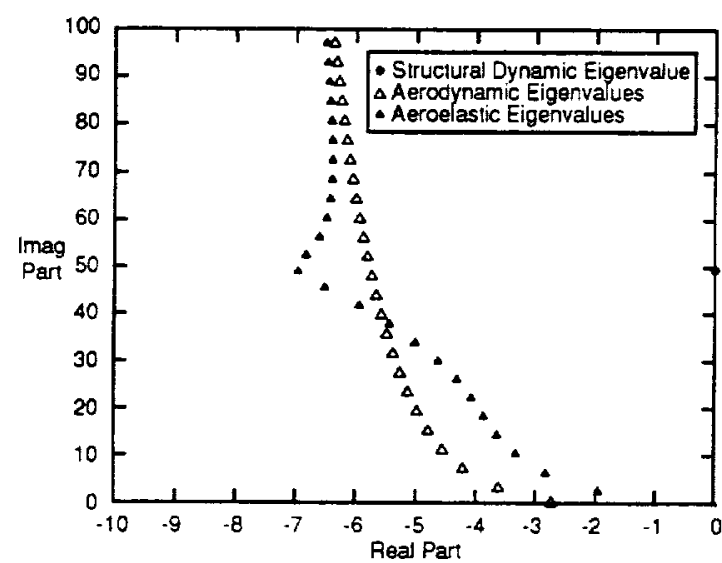

Figure 7 Eigenvalues for discretization with 10 aerodynamic elements on the wing, 1140 elements in the wake analyzed at the divergence reduced velocity, 3.8
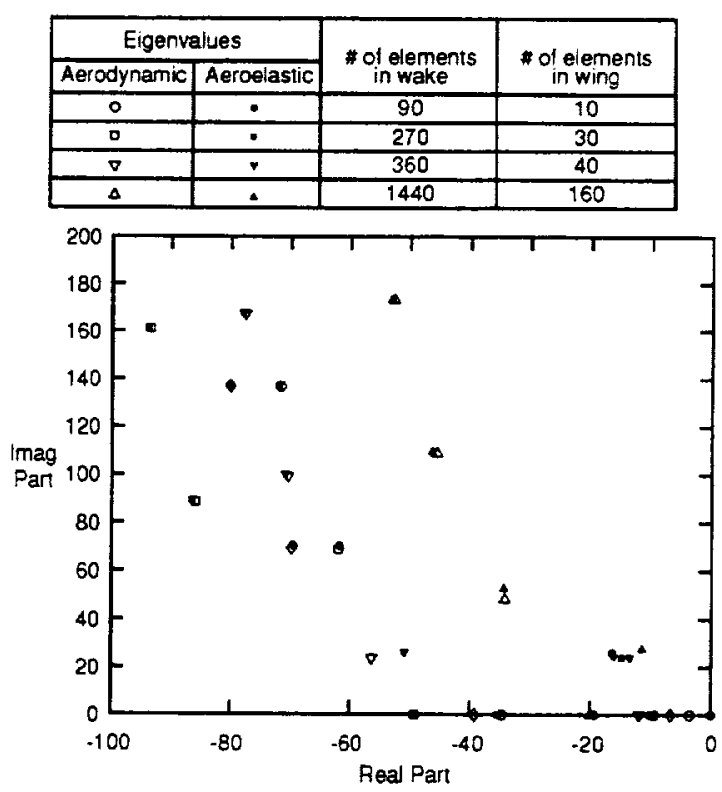

Figure 8. Eigenvalue patterns associated with aerodynamic discretizations with constant wake length of 9 chords analyzed at the divergence reduced velocity, 3.8

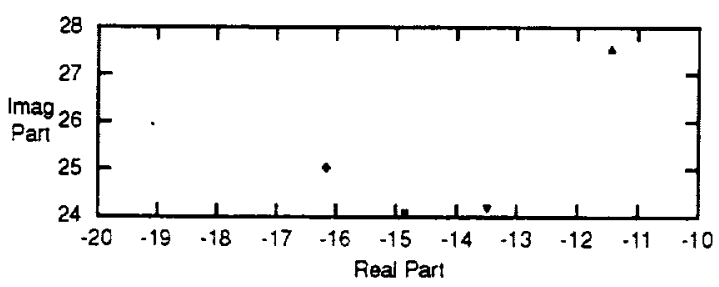

Figure 9 Enlargement of Figure 8; region near the structural dynamic originated eigenvalues

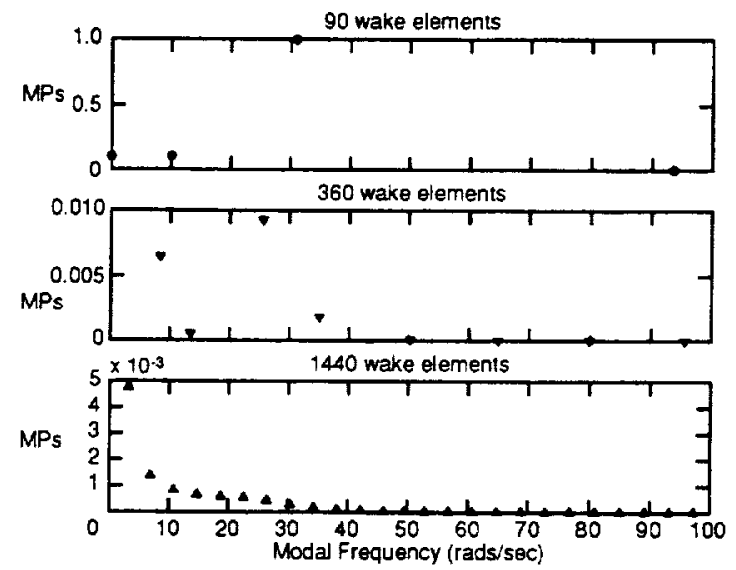

Figure 10 Structural modal participation factors

American Institute of Aeronautics and Astronautics 


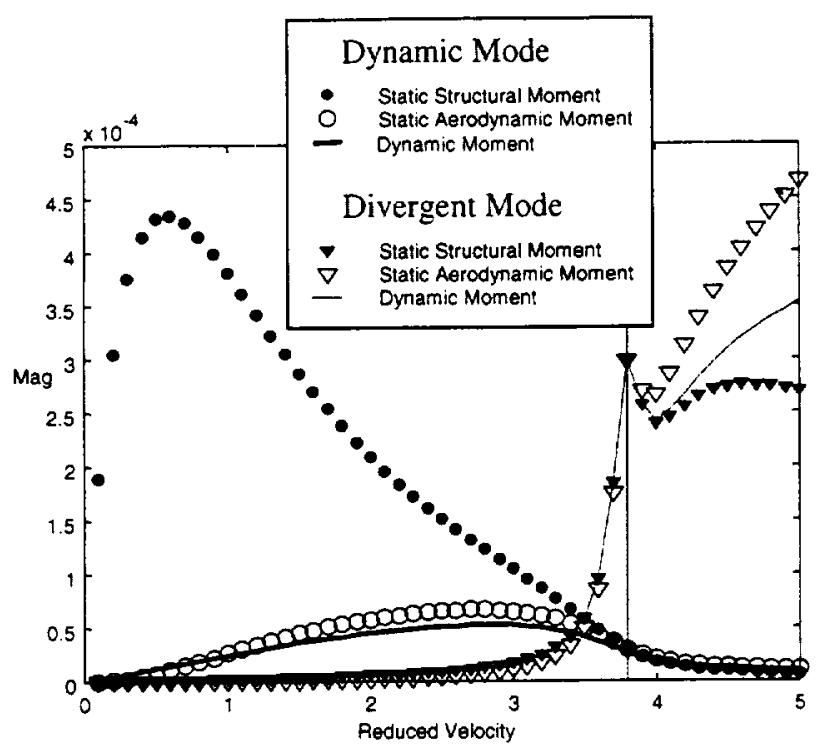

Figure 13 Modal moments associated with the structural-dynamic-originated mode and the divergent mode 


